\title{
Relevance of interferon-gamma in pathogenesis of life-threatening rapidly progressive interstitial lung disease in patients with dermatomyositis
}

Yuichi Ishikawa', Shigeru Iwata', Kentaro Hanami', Aya Nawata1,2, Mingzeng Zhang ${ }^{1}$, Kaoru Yamagata', Shintaro Hirata ${ }^{1,3}$, Kei Sakata ${ }^{1,4}$, Yasuyuki Todoroki ${ }^{1}$, Kazuhisa Nakano ${ }^{1}$, Shingo Nakayamada ${ }^{1}$, Minoru Satoh ${ }^{5}$ and Yoshiya Tanaka*

\begin{abstract}
Background: Dermatomyositis (DM) with rapidly progressive interstitial lung disease (DM RP-ILD) is a lifethreatening condition. Serum cytokine levels are potentially suitable biomarkers for DM RP-ILD. However, the relationships among cytokine levels, lung imaging findings, and lung pathology have not been investigated. The aim of the present retrospective study was to determine the association between hypercytokinemia and lung inflammation in patients with DM RP-ILD.

Methods: The study subjects were nine patients with life-threatening DM RP-ILD and severe hypoxemia (partial arterial oxygen pressure $\left(\mathrm{PaO}_{2}\right)$ /fraction of inspired oxygen $\left(\mathrm{FiO}_{2}\right)$ ratio $\leq 200$ ) before receiving intensive care management, who were admitted to our hospital between 2006 and 2015. The controls included 10 patients with DM without RP-ILD and 19 healthy subjects. We assessed the association between serum cytokine levels and computed tomography (CT) scores of the lung (ground glass opacity-score, G-score; fibrosis-score, F-score). Lung, hilar lymph nodes, and spleen from two autopsies were examined by hematoxylin-eosin (H\&E) staining and immunostaining.

Results: Serum interferon (IFN)- $\gamma$, interleukin (IL)-1 $\beta$ and IL-12 levels were significantly higher in patients with DM RP-ILD than in the other two groups, whereas serum IL-6 levels were elevated in the two patient groups but not in the healthy subjects. Serum levels of IL-2, IL-4, IL-8, IL-10, IFN-a, and TNF (tumor necrosis factor)-a were not characteristically elevated in the DM RP-ILD group. Serum IFN- $\gamma$ levels correlated with G-scores in patients with DM RP-ILD, while IL-1 $\beta$ was negatively correlation with F-scores. Immunohistochemical staining showed infiltration of numerous IFN- $\gamma$-positive histiocytes in the lung and hilar lymph nodes; but not in the spleen. Serum IL-6 levels did not correlate with the CT scores. Numerous IL-6-positive plasma cells were found in hilar lymph nodes, but not in the lungs or spleen.
\end{abstract}

Conclusions: Our results suggest strong IFN- - -related immune reaction in the lungs and hilar lymph nodes of patients with life-threatening DM RP-ILD, and potential IFN- $\gamma$ involvement in the pathogenesis of DM, specifically in the pulmonary lesions of RP-ILD.

Keywords: Rapidly progressive interstitial lung disease, Dermatomyositis, IFN- $\gamma$

\footnotetext{
* Correspondence: tanaka@med.uoeh-u.ac.jp

${ }^{1}$ The First Department of Internal Medicine, School of Medicine, University of Occupational and Environmental Health, Japan, 1-1 Iseigaoka, Yahatanishi-ku, Kitakyushu City 807-8555, Japan

Full list of author information is available at the end of the article
}

(c) The Author(s). 2018 Open Access This article is distributed under the terms of the Creative Commons Attribution 4.0 International License (http://creativecommons.org/licenses/by/4.0/), which permits unrestricted use, distribution, and reproduction in any medium, provided you give appropriate credit to the original author(s) and the source, provide a link to the Creative Commons license, and indicate if changes were made. The Creative Commons Public Domain Dedication waiver (http://creativecommons.org/publicdomain/zero/1.0/) applies to the data made available in this article, unless otherwise stated. 


\section{Background}

The rate of interstitial lung disease (ILD) in patients with dermatomyositis (DM) is approximately $30 \%[1,2]$. While most patients exhibit slow progression of ILD, some exhibit rapidly progressive ILD (RP-ILD), in which the respiratory status deteriorates rapidly within 2-3 months from the onset of ILD [3-5]. In particular, a high incidence of RP-ILD has been reported in patients with clinically amyopathic dermatomyositis (cADM) who are positive for anti-melanoma differentiation-associated gene 5 (MDA5) antibodies (Abs) $[6,7]$. RP-ILD in CADM is extremely difficult to treat and associated with a high mortality rate. Kameda et al. [8] reported the efficacy of intensive therapy with high-dose glucocorticoids (GC), intravenous cyclophosphamide (IVCY), and cyclosporine-A (CsA) in patients with DM complicated with RP-ILD (DM RP-ILD). Nakashima et al. [9] also reported marked improvement in prognosis of anti-MDA5 Abs-positive patients with DM using the same regimen, from the early stages of RP-ILD, with 75\% survival rate by intensive immunosuppressive regimen versus only about $29 \%$ by conventional step-up therapy. Despite these encouraging reports, poor prognosis has been reported even in patients on intensive therapy, such as those with anti-MDA5 Abs-positive cADM, with a mortality rate after 6 months of treatment of as high as $25 \%$ [8]. In a retrospective analysis of 56 patients (including 49 patients with RP-ILD) treated in the intensive care unit (ICU) for exacerbation of DM/polymyositis (PM), Peng et al. [10] reported an overall survival rate of $14 \%$ ( $n=8$ out of 56 ), though the survival rate after 28 days was $0 \%$ in patients with CADM. Thus, the prognosis of anti-MDA5 Abs-positive cADM patients with RP-ILD is poor, as is the prognosis of patients with DM who develop RP-ILD during the course of treatment. Although it has been reported that treatment with tacrolimus (TAC), a calcineurin inhibitor, similar to CsA, and rituximab (RTX), is effective for life-threatening DM RP-ILD refractory to the above intensive therapy [11-13], this outcome remains to be confirmed.

Almost all anti-MDA5 Abs-positive patients have cADM with a high incidence of acute or subacute ILD [6, 14]. In a retrospective analysis of 13 patients with anti-MDA5 Abs-positive cADM, Takada et al. [15] reported that mortality was associated with high levels of anti-MDA5 Abs, suggesting that the levels of anti-MDA5 Abs could be useful in predicting prognosis. Since a strong association between DM RP-ILD and anti-MDA5 Abs has been confirmed previously in several studies, research on the pathophysiology of DM RP-ILD has been conducted mainly in anti-MDA5 Abs-positive patients [16]. High serum levels of ferritin and several types of inflammatory cytokines have been described in patients with DM RP-ILD [17-21], suggesting their involvement in the pathogenesis of RP-ILD. The pathophysiology of DM RP-ILD could be similar to that of macrophage activation syndrome (MAS), in which a variety of cytokines (e.g., interleukin (IL)-1, IL-6, tumor necrosis factor (TNF)- $\alpha$ ) are involved [22]. However, despite studies suggesting that serum cytokines levels could be useful biomarkers for monitoring disease activity and to predict the prognosis of DM RP-ILD, the associations among serum cytokine levels, pulmonary image findings (e.g., computed tomography (CT) score) and lung pathology, have not been investigated thoroughly. The present study was designed to determine the relationships among serum cytokine levels, CT scores of the lung, and the histopathologic assessment of lung tissue.

\section{Methods}

\section{Study design and patients}

This study included nine Japanese patients with DM, aged $\geq 20$ years, who had life-threatening RP-ILD and were admitted to our department between 2006 and 2015 and treated at the in-patient intensive care management unit. The term RP-ILD is not well-established and is used mainly by rheumatologists but not by pneumologists. Since we understand that the lack of standardization of the term RP-ILD can cause clinical bias and confusion, we defined RP-ILD with reference to the definition of acute respiratory distress syndrome (ARDS) in this study [23, 24]. Life-threatening RP-ILD was defined based on previous reports $[7,23,24]$ as "a critical condition characterized by severe hypoxemia $\left(\mathrm{PaO}_{2} / \mathrm{FiO}_{2}\right.$ ratio $\left.\leq 200\right)$ that progressed within 3 months before initiation of treatment or intensification". The control groups included $10 \mathrm{pa}$ tients with DM with ILD (that did not meet the definition of RP-ILD) who underwent high-dose GC therapy (equivalent to prednisolone (PSL) of $>1 \mathrm{mg} / \mathrm{kg} /$ day) and 19 healthy individuals. Age-matched patients with DM without RP-ILD were randomly selected from the cohort of patients with DM/PM who were admitted to our department $(n=38)$ between 2014 and 2015. Thus, the total number of subjects in this study was 38 . With regard to evaluation of serum cytokines, the major cytokines (IL-1 $\beta$, IL-2, IL-4, IL-6, IL-8, IL-10, IL-12, interferon (IFN)- $\gamma$, IFN- $\alpha$, TNF- $\alpha$ ) involved in DM RP-ILD and MAS were selected based on the literature [17-22]. Cytokine levels were measured in all disease groups before the initiation or intensification of the treatment.

For patients with DM RP-ILD, the CT scores of the lung (ground glass opacity (GGO) score (G-score), fibrosis score (F-score)) and their association with serum cytokine levels were analyzed. This study was approved by the institutional review board of our university (\#H28-033).

\section{Diagnostic criteria}

The diagnosis of DM was based on the Bohan and Peter criteria for PM/DM while that of CADM was 
based on the diagnostic criteria of Euwer and Sontheimer [25-28].

\section{Exclusion criteria}

Patients with pulmonary lesions due to bacterial pneumonia, fungal pneumonia, or pneumocystis pneumonia (PCP) and those with sepsis were excluded. Bacterial pneumonia was diagnosed based on positive sputum culture and detection of bacteria phagocytosed by leukocytes. Fungal pneumonia was diagnosed based on positive sputum or bronchoalveolar lavage fluid (BALF) culture for fungi, high serum $\beta$-D-glucan levels, positivity for antigens of Candida, Aspergillus or Cryptococcus, and chest $\mathrm{CT}$ findings consistent with fungal pneumonia. PCP was diagnosed based on positive polymerase chain reaction (PCR) of the sputum or BALF for Pneumocystis jirovecii, and chest CT findings consistent with PCP. Sepsis was diagnosed based on The Third International Consensus Definitions for Sepsis and Septic Shock [29].

\section{$R P-I L D$ assessment by CT scores}

Lung CT was evaluated semi-quantitatively using two-types of CT score; the G-score, which reflects changes in the acute and active phases, and the F-score, which reflects changes mainly in the chronic phase. Images were scored by two rheumatologists with at least 15 years of clinical experience, who were blinded to the demographic and clinical information. The left and right lung fields were divided into three regions and total of six lung zones were scored separately: upper (aortic arch zone), middle (tracheal bifurcation zone), and lower (supradiaphragmatic zone). The G-score and F-score of each zone were scored on a scale of 0-3 (maximum score $=3$ points). The final CT score used for the analysis was the mean score of the six zones assessed by the two rheumatologists. The criteria used for the G-score were as follows: 1 point for predominantly subpleural partial GGO (Fig. 1A-a), 2 points for more pronounced GGO relative to that of G-score 1 point (Fig. 1A-b), and 3 points for diffuse GGO extending over a wide area (Fig. 1A-c). On the other hand, the criteria used for the F-score were as follows: 1 point for thickening and fibrosis of parts of the interlobular septa, mainly in the subpleural area (Fig. 1B-a), 2 points for more pronounced fibrosis and bronchiectasis compared with that for 1 point (Fig. 1B-b), and 3 points for diffuse and widespread fibrosis, honeycomb lung, and bronchiectasis (Fig. 1B-c) [30-32].

\section{Endpoints and clinical assessment}

The primary endpoint was elucidation of the significance of the elevated cytokines in DM RP-ILD. The secondary endpoint was the correlation between serum cytokines and CT scores in DM RP-ILD.

\section{Measurements of serum cytokine levels}

We measured the serum concentrations of various cytokines (IL-1 $\beta$, IL-2, IL-4, IL-6, IL-8, IL-10, IL-12, IFN- $\alpha$, IFN- $\gamma$, TNF- $\alpha$ ) at the time of admission. Serum samples were isolated and stored at $-80{ }^{\circ} \mathrm{C}$ until analysis. The concentrations of these cytokines were measured by cytometric bead array (Becton Dickinson, Franklin Lakes, NJ, USA) using a FACSVerse flow cytometer (Becton Dickinson). Data were analyzed using the FCAP Array software (Becton Dickinson).

\section{Immunohistochemical analysis}

Immunohistochemical analysis was performed as described previously [33]. Antigen retrieval was performed by soaking the specimen on slides in $5 \mathrm{M}$ sodium citrate solution in phosphate-buffered saline containing $0.05 \%$ $(v / v)$ Tween 20 (PBST) (pH 6.0). The slides were subsequently blocked with serum-free protein block (Dako, 2016-08) for $30 \mathrm{~min}$ at room temperature and incubated at $4{ }^{\circ} \mathrm{C}$ overnight optimally with rabbit polyclonal antibodies to IFN- $\gamma$ (ab25101, Abcam Inc., Boston, MA, USA) or IL-6 (21865-1-AP, Proteintech Inc., Rosemont, IL, USA) diluted 1:200 in DAKO antibody diluent. After washing three times with PBST, the slides were incubated with anti-rabbit IgG secondary antibody conjugated with horseradish peroxidase-labeled polymer (DakoCytomation, Glostrup, Denmark) and subsequently visualized by treatment with 3,3' diaminobenzidine (DAB) Chromogen (DakoCytomation, \#K3465) according to the instructions provided by the manufacturer. Nuclei were visualized using Mayer's hematoxylin (MERCK, 1:1000 dilution in PBST). For mounting, the sections were rinsed with water, dehydrated in graded ethanol (90\% ethanol for $30 \mathrm{~s} \times 3$ and $100 \%$ ethanol for $30 \mathrm{~s} \times 3$ ), cleared in xylene (for $30 \mathrm{~s} \times 2$ ), and sealed using multi-mount 480 (Matsunami, FM48001). Images were acquired and processed digitally.

\section{Measurement of myositis-specific autoantibodies (MSAs)}

We tested all serum samples by immunoprecipitation and enzyme-linked immunosorbent assay (ELISA) using recombinant proteins for anti-MDA5, anti-Jo-1, centromere protein A (CENP-A), CENP-B, Ro-52, and Ro-60 Abs. In addition, in patients positive for anti-aminoacyl-transfer ribonucleic acid synthetase (anti-ARS) Abs, the levels of anti-glycyl-tRNA synthetase (anti-EJ), anti-threonyl-tRNA synthetase (PL-7), anti-PL12, and anti-KS Abs were analyzed by ELISA.

\section{Immunoprecipitation}

Myositis-specific autoantibodies in serum were analyzed by immunoprecipitation of $\mathrm{K} 562$ cell extracts radiolabeled with ${ }^{35} \mathrm{~S}$-methionine as described previously [34]; the specificities of the autoantibodies were determined 


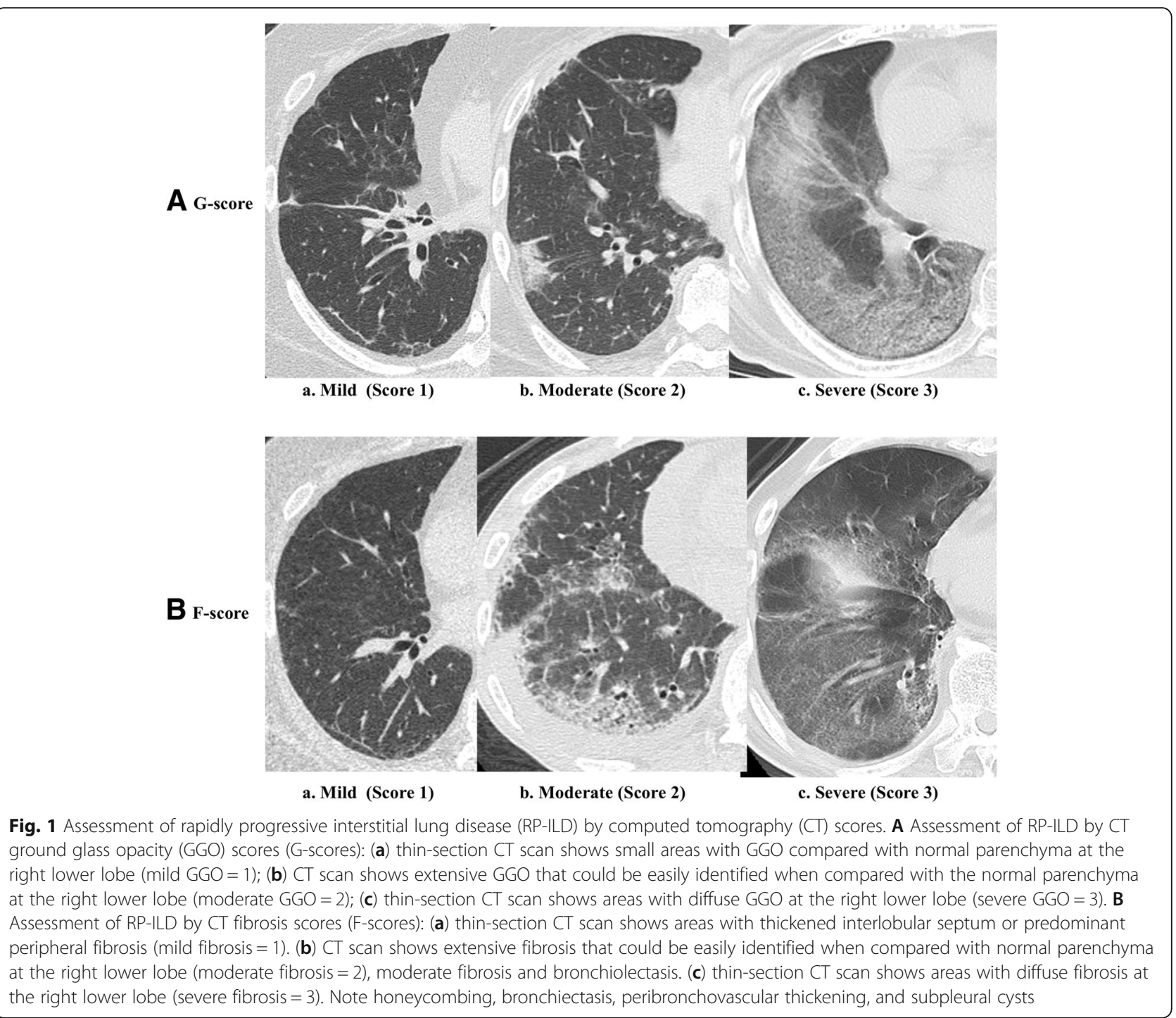

using specific reference serum. Analysis of RNA components of the immunoprecipitates was also performed when necessary.

\section{Anti-MDA5 and Jo-1 ELISA}

Anti-MDA5 and Jo-1 Abs were tested by enzyme-linked immunosorbent assay, using recombinant proteins $(0.5 \mu \mathrm{g} / \mathrm{ml}$; Diarect AG, Freiburg, Germany) and 1:250 diluted serum, as described previously [34,35]. The optical density was measured and converted into units using a standard curve created by a prototype-positive serum. The specificity of ELISA-positive serum was confirmed by immunoprecipitation.

\section{Statistical analysis}

Continuous variables were reported as mean plus or minus standard deviation or median (interquartile range). Differences between two groups were compared using the Mann-Whitney $U$ test. Differences among multiple groups were compared using the Kruskal-Wallis test, followed by post-hoc Dunn's multiple comparison test. Multiple group tests using the median test were also performed to determine the median values; the Wilcoxon test was used as the post-hoc test. The correlation between serum cytokine levels and CT scores was calculated using Spearman's correlation coefficient. Statistical significance was set at $p<0.05$. Statistical analyses were performed using the JMP version 9.0 (SAS Institute Inc., Cary, NC, USA).

\section{Results}

Demographic data of patients

The demographic data of patients in the DM RP-ILD groups are summarized in Table 1 . The disease duration 
Table 1 Clinical characteristics of patients

\begin{tabular}{|c|c|c|c|}
\hline & DM with RP-ILD & DM without RP-ILD & $p$ value \\
\hline $\mathrm{n}$ & 9 & 10 & \\
\hline Age, years & $69.3 \pm 3.9$ & $63.9 \pm 14.2$ & 0.68 \\
\hline Female $(n, \%)$ & $8,88.9$ & $6,60.0$ & 0.31 \\
\hline $\begin{array}{l}\text { Disease duration } \\
\text { (months) }\end{array}$ & $18.1 \pm 39.8$ & $7.6 \pm 8.7$ & 0.40 \\
\hline $\begin{array}{l}\text { Smokers (current } \\
\text { and past) (\%) }\end{array}$ & 11.1 & 30.0 & 0.31 \\
\hline $\begin{array}{l}\text { Number of GC } \\
\text { pulses }\end{array}$ & $2.2 \pm 1.1$ & N/A & \\
\hline $\mathrm{PaO}_{2} / \mathrm{FiO}_{2}$ ratio & $160 \pm 90$ & N/A & \\
\hline $\begin{array}{l}\text { Leukocyte } \\
\text { count }(/ \mu \mathrm{L})\end{array}$ & $9438 \pm 5751$ & $7620 \pm 5458$ & 0.35 \\
\hline $\mathrm{LDH}(\mathrm{U} / \mathrm{L})$ & $549 \pm 357$ & $376 \pm 168$ & 0.27 \\
\hline $\mathrm{KL}-6(\mathrm{U} / \mathrm{mL})$ & $1087 \pm 584$ & $1419 \pm 1756$ & 0.46 \\
\hline $\operatorname{lgG}(\mathrm{mg} / \mathrm{dL})$ & $1225 \pm 398$ & $1452 \pm 454$ & 0.27 \\
\hline $\begin{array}{l}\text { Positivity for anti- } \\
\text { CADM140/MDA5 Ab (\%) }\end{array}$ & 66.7 & 50.0 & 0.76 \\
\hline CT score $(\mathrm{G})$ & $2.1 \pm 0.7$ & N/A & \\
\hline CT score $(\mathrm{F})$ & $1.2 \pm 0.6$ & N/A & \\
\hline
\end{tabular}

Data are mean $\pm S D$ or number of patients (percentage)

$D M$ dermatomyositis, $R P$-ILD rapidly progressive-interstitial lung disease, $G \mathrm{C}$ glucocorticoid, $\mathrm{PaO}_{2} / \mathrm{FiO}_{2}$ partial arterial pressure of oxygen/fraction of inspired oxygen, KL- 6 Kerbs von Lungren 6 antigen, $C T$ computed tomograpghy, $G$ ground glass opacity, $F$ fibrosis

of DM with and without RP-ILD was $18.1 \pm 39.8$ and 7.6 \pm 8.7 months, respectively (Table 1 ). Among the 19 patients, 11 were considered to have new-onset untreated anti-MDA5 Abs-positive DM. All six anti-MDA5 antibody-positive patients with DM with RP-ILD had hypoxemia (partial arterial pressure of oxygen $\left(\mathrm{PaO}_{2}\right) /$ fraction of inspired oxygen $\left(\mathrm{FiO}_{2}\right)$ ratio $\left.\leq 200\right)$ before starting intensive therapy and their disease duration was $1.2 \pm$ 0.4 months. On the other hand, none of the five anti-MDA5 antibody-positive patients with DM who were free of RP-ILD were hypoxemic before the start of treatment, and their disease duration was $5.5 \pm 4.3$ months.

The DM RP-ILD group included two patients positive for anti-PL-7 Ab (Additional file 1: Table S1), who developed RP-ILD during the course of remission maintenance therapy and thus, had long disease duration (35 and 120 months). One patient was treated with $7.5 \mathrm{mg} /$ day PSL and the other with $3 \mathrm{mg} /$ day TAC. The disease duration was long in the DM with RP-ILD group because this group not only included anti-MDA5 Abs-positive patients but also two anti-PL-7 antibody-positive patients.

High serum IFN- $\gamma, \mathrm{IL}-1 \beta$, and IL-12 levels in patients with DM RP-ILD

Figure 2 and Additional file 2: Table S2 compare serum cytokine levels among the DM RP-ILD, DM without
RP-ILD, and HD groups, while Additional file 1: Table S1 shows antibody profiles and serum cytokine profiles in the same three groups. Serum levels of IFN- $\gamma$, IL-1 $\beta$, and IL-12 were significantly higher in the DM RP-ILD group compared with the other two groups (IFN- $\gamma, p<0.01$ vs DM without RP-ILD, and $p<0.01$ vs healthy donors (HD); IL- $1 \beta, p=0.03$ vs DM without RP-ILD, and $p<0.01$ vs HD; IL-12, $p<0.01$ vs DM without RP-ILD and $p<0.01$ vs HD). Furthermore, serum levels of IL-6, IL-10, and IFN- $\alpha$ were significantly higher in the DM RP-ILD group compared with the healthy donors, but were not significantly different from those in the DM without RP-ILD group. Interestingly, the serum levels of IL-2, IL-4, IL-8, and TNF- $\alpha$ levels were within the normal ranges in the DM RP-ILD group (Fig. 2). These results suggest that high serum levels of IFN- $\gamma$, IL-1 $\beta$, and IL-12 are characteristic of DM RP-ILD. Unlike previous studies [17, 20, 21], our results showed no characteristic rises in IL-6, IL-8, IL-10, IFN- $\alpha$, and TNF- $\alpha$ in DM RP-ILD.

\section{Serum IFN- $\gamma$ levels correlate significantly with G-score in patients with DM RP-ILD}

In addition to the high serum levels of IFN- $\gamma$ (Fig. 2), in the DM RP-ILD group there was positive correlation between serum IFN- $\gamma$ levels and the G-scores $(\rho=0.69, p=0.04$, Table 2). Although serum IL-1 $\beta$ also correlated significantly with the F-scores, the correlation was negative $(\rho=-0.68, p=0.045$, Table 2$)$. None of the other cytokines were significantly correlated with the CT scores. These results demonstrate characteristically high serum IFN- $\gamma$ in patients with DM RP-ILD, and significant correlation between IFN- $\gamma$ and the G-score, which is a marker of the acute phase and disease activity in ILD. Moreover, the results suggest that IFN- $\gamma$ plays a major role in the pathophysiology of DM RP-ILD.

\section{Accumulation of IFN- $\gamma$-positive histiocytes in lungs and hilar lymph nodes, and IL-6-positive plasma cells in hilar lymph nodes in patients with DM RP-ILD}

Serum IFN- $\gamma$ was characteristically high and correlated with the G-scores in the DM RP-ILD group, whereas serum IL-6 was not elevated characteristically and did not correlate with the CT scores (Fig. 2 and Table 2), although IL-6 is reported to be important in DM RP-ILD $[17,19,36,37]$. In the next step, we examined the roles of IFN- $\gamma$ and IL-6 in the pulmonary pathophysiology of DM RP-ILD by immunostaining and hematoxylin-eosin (H\&E) staining of lung tissues, hilar lymph nodes, and spleen tissues from two patients from whom specimens were obtained on autopsy (Fig. 3).

The first patient (Case 1) was a 70-year-old man with anti-MDA5 antibody-positive cADM. The patient was treated with four courses of GC pulse therapy, TAC, 


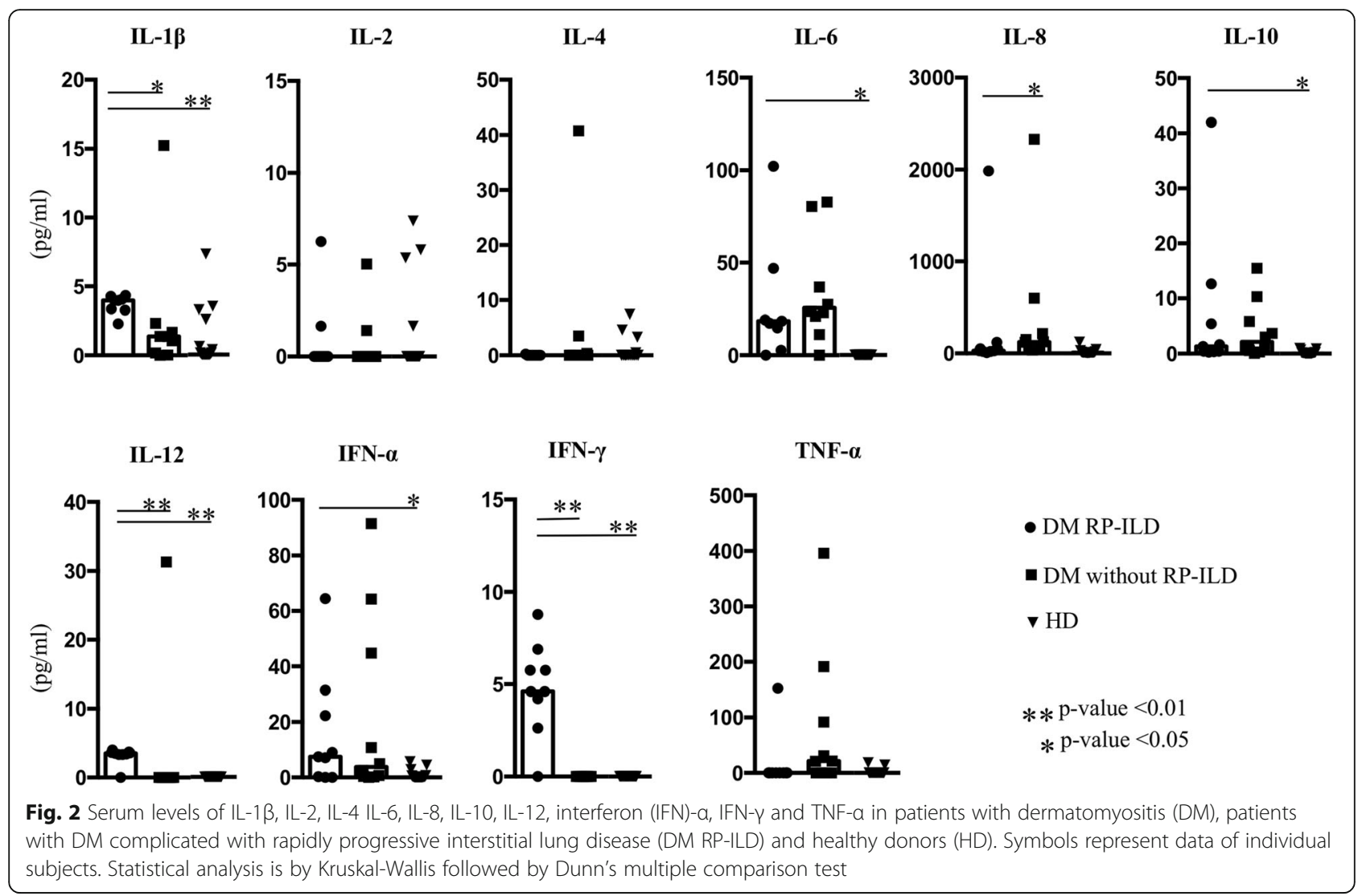

CsA, IVCY, and RTX (Fig. 3a). H\&E staining showed diffuse hyaline membrane formation in the alveolar spaces. Fibroblast proliferation and incorporation of this hyaline membrane were observed in some parts, suggesting diffuse alveolar damage (DAD) extending from the exudative phase to the organizing phase (Fig. 3a (1)). Further analysis showed extravasation of erythrocytes and infiltration and aggregation of histiocytes in the alveolar spaces (Fig. 3a (2)). In these same tissues, IFN- $\gamma$-stained histiocytes had abundant cytoplasm and eccentrically distributed large nuclei (Fig. 3a (3, 4), red arrows). Marked infiltration of histiocytes was also noted into the lymph sinus of the hilar lymph nodes, with disappearance of nearly all lymphoid follicles (Fig. 3a $(5,6)$ ). In addition to the lung tissue, IFN- $\gamma$-stained histiocytes were also found in the hilar lymph nodes (Fig. 3a $(7,8)$, histiocytes marked by red arrows). However, H\&E staining of the spleen showed no marked histopathological changes other than splenic white pulp atrophy (Fig. 3a $(9,10))$, with no infiltration of IFN- $\gamma$-positive histiocytes (Fig. 3a $(11,12))$. The results of IL-6 staining are shown in Fig. 3a $(13,14,15,16,17,18)$. In the lung and spleen tissues, a few IL-6-positive histiocytes were observed, but cytoplasmic immunostaining was relatively weak
(Fig. 3a (13, 14, 17, 18), histiocytes marked by the red arrow). Numerous IL-6-positive plasma cells were observed in hilar lymph nodes (Fig. 3a $(15,16)$, plasma cells marked by blue arrows). A small number of IL-6-positive histiocytes and plasma cells were also found in the spleen (Fig. 3a (17, 18), histiocytes and plasma cells are marked by the red and blue arrows).

The other autopsy specimen was from a 65-year-old woman with anti-PL-7 antibody-positive DM treated with one course of GC pulse therapy and IVCY (Fig. 3b). At the onset of RP-ILD, she was treated with $7.5 \mathrm{mg} /$ day PSL, followed by a course of GC pulse therapy, TAC, and IVCY. The histopathological findings were similar to those of the first patient, despite different treatment histories and types of MSA. These results suggest that the pathophysiology of DM RP-ILD seems to be characterized by local appearance of IFN- $\gamma$-positive histiocytes in the lung tissues and related lymphoid tissues and the appearance of IL-6-positive plasma cells in hilar lymph nodes, regardless of the treatment history and type of MSA.

\section{Discussion}

The present study demonstrated the presence of characteristically high serum IFN- $\gamma$ in patients with life-threatening 
Table 2 Association between CT scores and cytokines in patients with DM RP-ILD

\begin{tabular}{|c|c|c|}
\hline & $\rho$ & $p$ value \\
\hline \multicolumn{3}{|c|}{ CT scores (F) } \\
\hline IFN- $\gamma$ & 0.10 & 0.80 \\
\hline$I L-1 \beta$ & -0.68 & 0.05 \\
\hline IL-6 & 0.35 & 0.36 \\
\hline IL-12 & -0.14 & 0.71 \\
\hline TNF-a & -0.43 & 0.25 \\
\hline $\mid \mathrm{L}-2$ & -0.17 & 0.67 \\
\hline $\mid \mathrm{L}-4$ & -0.07 & 0.85 \\
\hline IL-8 & -0.56 & 0.12 \\
\hline IL-10 & -0.49 & 0.18 \\
\hline IFN-a & -0.15 & 0.70 \\
\hline \multicolumn{3}{|c|}{ CT scores (G) } \\
\hline IFN- $\gamma$ & 0.69 & 0.04 \\
\hline$I L-1 \beta$ & 0.14 & 0.72 \\
\hline IL-6 & 0.24 & 0.53 \\
\hline IL-12 & 0.10 & 0.80 \\
\hline TNF-a & -0.21 & 0.59 \\
\hline $\mathrm{IL}-2$ & -0.45 & 0.22 \\
\hline IL-4 & 0.07 & 0.86 \\
\hline $\mid \mathrm{L}-8$ & -0.12 & 0.76 \\
\hline IL-10 & -0.53 & 0.14 \\
\hline IFN-a & -0.35 & 0.35 \\
\hline
\end{tabular}

$D M$ dermatomyositis, $R P$-ILD rapidly progressive-interstitial lung disease, CT computed tomography, G ground glass opacity, F fibrosis, IFN interferon, IL interleukin, TNF tumor necrosis factor

DM RP-ILD and that such levels correlated significantly with CT scores and histopathologic findings of pulmonary lesions. While high serum IL-6 reported in previous studies was also observed in patients with DM without RP-ILD, this finding might not be a characteristic of DM RP-ILD. Our results also showed significant correlation between serum IFN- $\gamma$ levels and CT scores/G-scores, and infiltration of IFN- $\gamma$-positive histiocytes into the lung and hilar lymph node tissues, but not in the spleen, in patients with high disease activity. Numerous IL-6-positive plasma cells were also observed in the hilar lymph nodes but not in the lung. In the DM RP-ILD group, serum IFN- $\gamma$ was elevated even in anti-MDA5 antibody-negative cases, whereas in the DM without RP-ILD group, serum IFN- $\gamma$ was not elevated even in the majority of anti-MDA5 antibody-positive cases (Additional file 1: Table S1 and Additional file 2: Table S2). These results suggest that high serum IFN- $\gamma$ is associated with the onset of RP-ILD, regardless of the presence of anti-MDA5 antibodies in patients with DM. Gono et al. [19] reported that anti-MDA5 antibody-positive ILD patients with high disease activity and poor prognosis tend to have a low IL-4/IFN- $\gamma$ ratio, relative to patients with anti-ARS antibody-positive DM complicated with ILD. Considered together, these findings highlight the potential role of IFN- $\gamma$ in the pathophysiology of anti-MDA5 antibody-positive DM.

No common pathophysiological processes between MAS and DM RP-ILD have previously been described. MAS is a secondary hemophagocytic syndrome (HPS) or hemophagocytic lymphohistiocytosis (i.e., autoimmuneassociated HPS), in which various vital organs are damaged due to abnormal production of pro-inflammatory cytokines, such as IFN- $\gamma$ [38]. It has been reported that hyperferritinemia, which reflects macrophage activation, is observed in $82 \%$ of patients with MAS [39, 40]. Moreover, cytopenia and liver dysfunction were often observed in patients with MAS $[39,41]$. On the other hand, serum ferritin levels correlated with disease activity in patients with anti-MDA5 antibody-positive DM complicated with RP-ILD [6], who often have liver dysfunction and cytopenia [6, 42, 43]. We identified high serum IFN- $\gamma$ and the presence of IFN- $\gamma$-positive histiocytes in the lung in patients with DM RP-ILD. These results suggest that in addition to its importance in MAS, IFN- $\gamma$ seems to have a pathological influence in DM RP-ILD by activating macrophages and accelerating inflammation. This is the first study to report the characteristic presence of high serum IFN- $\gamma$ in DM RP-ILD and that these levels correlate with the severity of pulmonary lesions assessed by CT scores/G-scores and histopathological examination.

In DM, serum IL-6 levels correlate with disease activity [44], and the use of tocilizumab (TCZ) is effective in patients with refractory DM [45]. However, there is no information on whether TCZ is effective against DM complicated with ILD or DM RP-ILD. Our study showed that (1) serum IL-6 was not specifically high only in DM RP-ILD but also in patients with DM without RP-ILD; (2) unlike IFN- $\gamma$, high serum IL-6 did not correlate with CT scores; and (3) numerous IL-6-positive plasma cells were found in hilar lymph nodes but not in the lungs. These results suggest that while IL-6 is important in the pathogenesis of DM RP-ILD, it is unlikely to be involved in local lung injury. Although serum IL- $1 \beta$ levels also correlated significantly with F-scores, the correlation was negative. Correlation between serum IL-1 $\beta$ levels and disease activity and pulmonary lesions was examined in previous studies, but no significant correlation was detected [17]. Our results also showed no significant correlation among other cytokines and CT scores in DM RP-ILD.

We expected to find systemic autoimmune inflammation, particularly in secondary lymphoid tissues, such as the spleen, in patients with DM RP-ILD. 


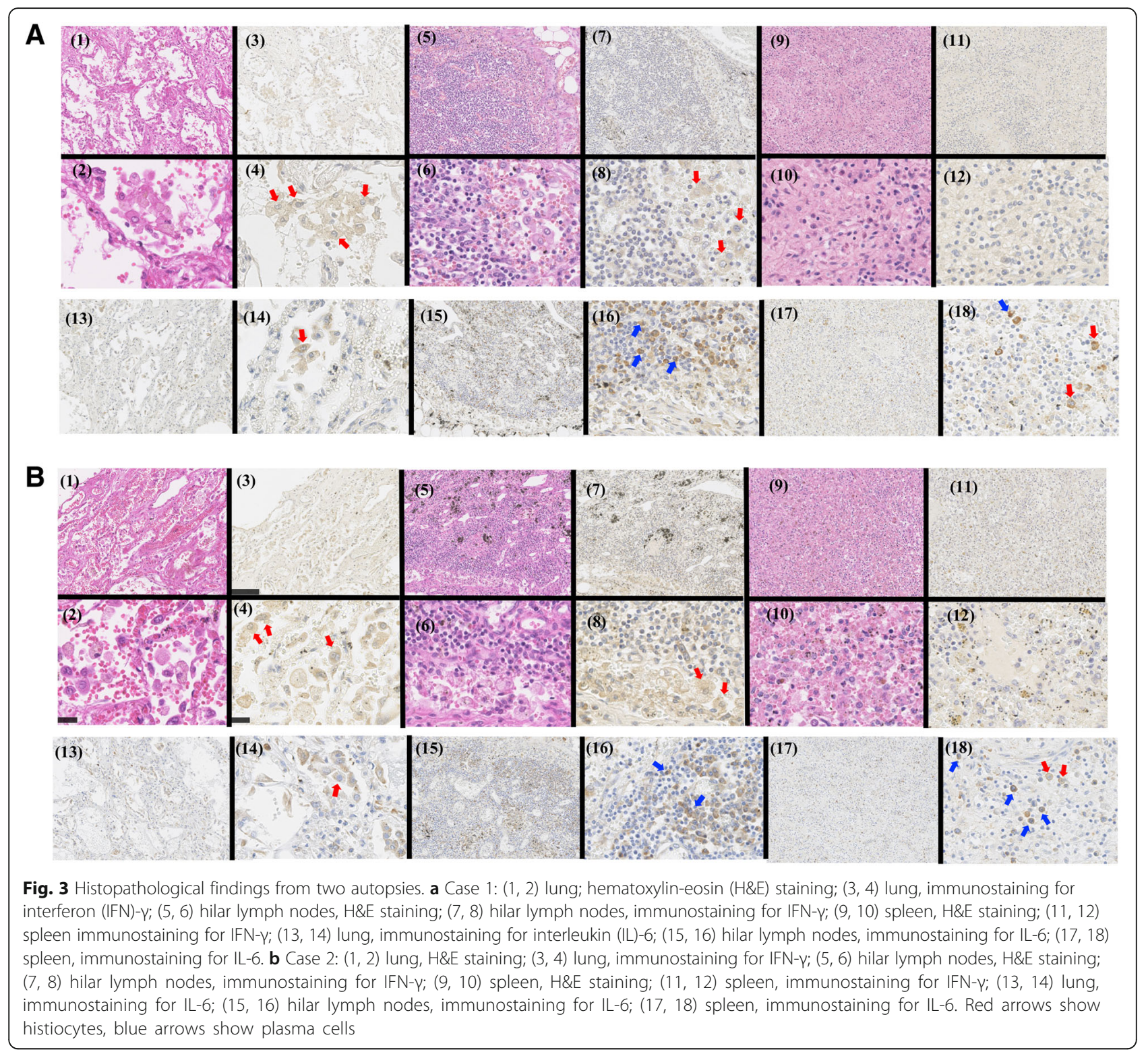

However, contrary to our expectation, the presence of IFN- $\gamma$-positive histiocytes was limited to local regions of the lungs and pulmonary hilar lymph nodes showing diffuse DAD. Neither infiltration of IFN- $\gamma$-positive histiocytes nor histopathological changes suggestive of inflammation were observed in the spleen. Inflammation limited to localized regions of the lungs may be a significant finding that could influence the selection of the drug administration route in the future.

The present study has certain limitations. First, the number of enrolled patients was relatively small because RP-ILD is an uncommon disease. Thus, our findings need to be confirmed in larger cohort studies. Second, it is possible that immunosuppressive therapy itself altered serum cytokine levels in the present study.

\section{Conclusions}

IFN- $\gamma$ was characteristically high in patients with DM RP-ILD after the onset of life-threatening RP-ILD. Furthermore, serum IFN- $\gamma$ levels correlated with GGO, as evaluated by CT. Our results also suggested that inflammation might occur in localized regions of the lungs. Considered together, the results suggest the high potential of IFN $-\gamma$ involvement in the pathophysiology of DM, specifically in the formation of pulmonary lesions seen in RP-ILD. Further prospective studies in large numbers of patients are needed. 


\section{Additional files}

Additional file 1: Table S1 Serum levels of various cytokines, antibody profiles, and treatment at the time of registration. (DOCX $27 \mathrm{~kb}$ )

Additional file 2: Table S2 Median values and ranges of the measured cytokines. (DOCX 16 kb)

\section{Abbreviations}

Abs: Antibodies; ARDS: Acute respiratory distress syndrome; ARS: Aminoacyltransfer ribonucleic acid synthetase; BALF: Bronchoalveolar lavage fluid; CADM: Clinically amyopathic dermatomyositis; CsA: Cyclosporine-A; CT: Computed tomography; DAD: Diffuse alveolar damage; DM RP-ILD: Dermatomyositis with rapidly progressive interstitial lung disease; DM: Dermatomyositis; ELISA: Enzymelinked immunosorbent assay; $\mathrm{FiO}_{2}$ : Fraction of inspired oxygen; F-score: Fibrosis score; GC: Glucocorticoid; GGO: Ground glass opacity; G-score: Ground glass opacity score; H\&E: Hematoxylin-eosin; HD: Healthy donors; ICU: Intensive care unit; IFN-a: Interferon alpha; IFN-y: Interferon gamma; IL: Interleukin; IL-1ß: Interleukin-1beta; ILD: Interstitial lung disease; IVCY: Intravenous cyclophosphamide; MAS: Macrophage activation syndrome; MDA5: Melanoma differentiation-associated gene 5; MSA: Myositis-specific antibody; $\mathrm{PaO}_{2}$ : Partial pressure of arterial oxygen; PCP: Pneumocystis pneumonia; PM: Polymyositis; PSL: Prednisolone; RNA: Ribonucleic acid; RP-ILD: Rapidly progressive interstitial lung disease; RTX: Rituximab; TAC: Tacrolimus; TNF-a: Tumor necrosis factor-alpha

\section{Acknowledgements}

The authors acknowledge the technical assistance and expertise of Ms Narumi Sakaguchi, Ms Kahoru Noda, Ms Tomoko Hasegawa, and Dr Shin Tanaka (University of Occupational and Environmental Health, Kitakyushu, Japan).

\section{Funding}

This work was supported in part by a Grant-In-Aid for Scientific Research from the Ministry of Education, Culture, Sports, Science, and Technology of Japan (\#22249025, \#16 K09928, \#15 K08790), the Ministry of Health, Labor, and Welfare of Japan (\#-126-008), and Japan Agency for Medical Research and Development (\#16ek0410016h0003).

\section{Authors' contributions}

$\mathrm{YI}, \mathrm{SI}, \mathrm{KH}, \mathrm{SN}, \mathrm{KN}$, and $\mathrm{YT}$ conceived the design of the study. $\mathrm{YI}$ and $\mathrm{YT}$ acquired the clinical data. SI, $\mathrm{KH}$, and $\mathrm{SH}$ evaluated the $\mathrm{CT}$ scores. $\mathrm{KH}$ and $\mathrm{SI}$ performed statistical analyses. YI, SI, and $\mathrm{KH}$ interpreted the data. KS and MZ measured serum cytokines. MS tested MSA. AN and KY performed histopathological examination. YI drafted the manuscript. All authors revised the manuscript for intellectual content and approved the final version.

\section{Ethics approval and consent to participate}

Ethical approval was obtained from the University of Occupational and Environmental Health, Japan Ethics Committee. This retrospective study was approved by the institutional review board, and the requirement to obtain informed consent was waived.

\section{Consent for publication}

Not applicable.

\section{Competing interests}

Kei Sakata is an employee of Mitsubishi Tanabe Pharma. Shingo Nakayamada has received speaking fees from Bristol-Myers, Sanofi, AbbVie, Eisai, Eli Lilly, Chugai, Pfizer, Takeda (less than US \$10,000 each), and research grants from Mitsubishi-Tanabe, Novartis and MSD. Dr Tanaka has received consulting fees, speaking fees, and/or honoraria from Mitsubishi Tanabe Pharma, Eisai, Pfizer, Abbott Immunology, Janssen, Takeda Industrial Pharma, Santen, AstraZeneca, Astellas, Asahi Kasei, UCB, and GlaxoSmithKline (less than US \$10,000 each) and from AbbVie and Chugai (more than US \$10,000 each) and research grants from Bristol-Myers Squibb, Mitsubishi Tanabe, MSD, Takeda Industrial Pharma, Astellas, Eisai, Chugai, Pfizer, and Daiichi-Sankyo. All other authors declare no conflict of interest.

\section{Publisher's Note}

Springer Nature remains neutral with regard to jurisdictional claims in published maps and institutional affiliations.

\section{Author details}

${ }^{1}$ The First Department of Internal Medicine, School of Medicine, University of Occupational and Environmental Health, Japan, 1-1 Iseigaoka, Yahatanishi-ku, Kitakyushu City 807-8555, Japan. ²Department of Pathology and Cell Biology, School of Medicine, University of Occupational and Environmental Health, Japan, Kitakyushu City, Japan. ${ }^{3}$ Department of Clinical Immunology and Rheumatology, Hiroshima University Hospital, Hiroshima, Japan. ${ }^{4}$ Mitsubishi Tanabe Pharma Corporation, Yokohama, Japan. ${ }^{5}$ Department of Clinical Nursing, School of Health Sciences, University of Occupational and Environmental Health, Japan, Kitakyushu City, Japan.

Received: 19 June 2018 Accepted: 28 September 2018

Published online: 26 October 2018

\section{References}

1. Ikezoe J, Johkoh T, Kohno N, Takeuchi N, Ichikado K, Nakamura H. Highresolution $C T$ findings of lung disease in patients with polymyositis and dermatomyositis. J Thorac Imaging. 1996;11(4):250-9.

2. Fathi M, Vikgren J, Boijsen M, Tylen $U$, Jorfeldt L, Tornling $G$, et al. Interstitial lung disease in polymyositis and dermatomyositis: Iongitudinal evaluation by pulmonary function and radiology. Arthritis Rheum. 2008;59(5):677-85.

3. Fathi $M$, Lundberg IE. Interstitial lung disease in polymyositis and dermatomyositis. Curr Opin Rheumatol. 2005;17(6):701-6.

4. Bouros D, Nicholson AC, Polychronopoulos V, du Bois RM. Acute interstitial pneumonia. Eur Respir J. 2000;15(2):412-8.

5. Al-Hameed FM, Sharma S. Outcome of patients admitted to the intensive care unit for acute exacerbation of idiopathic pulmonary fibrosis. Can Respir J. 2004;11(2):117-22.

6. Nakashima R, Imura Y, Kobayashi S, Yukawa N, Yoshifuji H, Nojima T, et al. The RIG-I-like receptor IFIH1/MDA5 is a dermatomyositis-specific autoantigen identified by the anti-CADM-140 antibody. Rheumatology (Oxford). 2010;49(3):433-40.

7. Sato $S$, Hirakata M, Kuwana M, Suwa A, Inada S, Mimori $T$, et al. Autoantibodies to a 140-kd polypeptide, CADM-140, in Japanese patients with clinically amyopathic dermatomyositis. Arthritis Rheum. 2005;52(5):1571-6.

8. Kameda $\mathrm{H}$, Nagasawa $\mathrm{H}$, Ogawa $\mathrm{H}$, Sekiguchi $\mathrm{N}$, Takei $\mathrm{H}$, Tokuhira $\mathrm{M}$, et al. Combination therapy with corticosteroids, cyclosporin A, and intravenous pulse cyclophosphamide for acute/subacute interstitial pneumonia in patients with dermatomyositis. J Rheumatol. 2005;32(9):1719-26.

9. Nakashima R, Hosono $Y$, Mimori T. Clinical significance and new detection system of autoantibodies in myositis with interstitial lung disease. Lupus. 2016;25(8):925-33

10. Peng JM, Du B, Wang Q, Weng L, Hu XY, Wu CY, et al. Dermatomyositis and polymyositis in the intensive care unit: a single-center retrospective cohort study of 102 patients. PLoS One. 2016;11(4):e0154441.

11. Kurita T, Yasuda S, Amengual O, Atsumi T. The efficacy of calcineurin inhibitors for the treatment of interstitial lung disease associated with polymyositis/dermatomyositis. Lupus. 2015;24(1):3-9.

12. Watanabe $R$, Ishii T, Araki $K$, Ishizuka M, Kamogawa $Y$, Fujita $Y$, et al. Successful multi-target therapy using corticosteroid, tacrolimus, cyclophosphamide, and rituximab for rapidly progressive interstitial lung disease in a patient with clinically amyopathic dermatomyositis. Mod Rheumatol. 2016;26(3):465-6.

13. Kurita T, Yasuda S, Oba K, Odani T, Kono M, Otomo K, et al. The efficacy of tacrolimus in patients with interstitial lung diseases complicated with polymyositis or dermatomyositis. Rheumatology (Oxford). 2015;54(1):39-44.

14. Sato $S$, Hoshino $K$, Satoh T, Fujita T, Kawakami $Y$, Kuwana M. RNA helicase encoded by melanoma differentiation-associated gene 5 is a major autoantigen in patients with clinically amyopathic dermatomyositis: association with rapidly progressive interstitial lung disease. Arthritis Rheum 2009;60(7):2193-200.

15. Takada T, Aoki A, Asakawa K, Sakagami T, Moriyama H, Narita I, et al. Serum cytokine profiles of patients with interstitial lung disease associated with anti-CADM-140/MDA5 antibody positive amyopathic dermatomyositis. Respir Med. 2015;109(9):1174-80. 
16. Abe Y, Matsushita M, Tada K, Yamaji K, Takasaki Y, Tamura N. Clinical characteristics and change in the antibody titres of patients with antiMDA5 antibody-positive inflammatory myositis. Rheumatology (Oxford) 2017;56(9):1492-7.

17. Kawasumi H, Gono T, Kawaguchi $Y$, Kaneko H, Katsumata $Y$, Hanaoka M, et al. IL-6, IL-8, and IL-10 are associated with hyperferritinemia in rapidly progressive interstitial lung disease with polymyositis/dermatomyositis. Biomed Res Int. 2014;2014(8):15245.

18. Gono T, Sato S, Kawaguchi Y, Kuwana M, Hanaoka M, Katsumata Y, et al. Anti-MDA5 antibody, ferritin and IL-18 are useful for the evaluation of response to treatment in interstitial lung disease with anti-MDA5 antibodypositive dermatomyositis. Rheumatology (Oxford). 2012;51(9):1563-70.

19. Gono T, Kawaguchi Y, Hara M, Masuda I, Katsumata Y, Shinozaki M, et al. Increased ferritin predicts development and severity of acute interstitial lung disease as a complication of dermatomyositis. Rheumatology (Oxford). 2010;49(7):1354-60.

20. Gono T, Kaneko H, Kawaguchi Y, Hanaoka M, Kataoka S, Kuwana M, et al. Cytokine profiles in polymyositis and dermatomyositis complicated by rapidly progressive or chronic interstitial lung disease. Rheumatology (Oxford). 2014;53(12):2196-203.

21. Horai Y, Koga T, Fujikawa K, Takatani A, Nishino A, Nakashima Y, et al. Serum interferon-a is a useful biomarker in patients with anti-melanoma differentiation-associated gene 5 (MDA5) antibody-positive dermatomyositis. Mod Rheumatol. 2015;25(1):85-9.

22. Schulert GS, Grom AA. Macrophage activation syndrome and cytokinedirected therapies. Best Pract Res Clin Rheumatol. 2014;28(2):277-92.

23. Ranieri VM, Rubenfeld GD, Thompson BT, Ferguson ND, Caldwell E, Fan E, et al. Acute respiratory distress syndrome: the Berlin Definition. JAMA. 2012; 307(23):2526-33.

24. Fan E, Brodie D, Slutsky AS. Acute respiratory distress syndrome: advances in diagnosis and treatment. JAMA. 2018;319(7):698-710.

25. Bohan A, Peter JB. Polymyositis and dermatomyositis (first of two parts). N Engl J Med. 1975;292(7):344-7.

26. Bohan A, Peter JB. Polymyositis and dermatomyositis (second of two parts). N Engl J Med. 1975;292(8):403-7.

27. Euwer RL, Sontheimer RD. Amyopathic dermatomyositis: a review. J Invest Dermatol. 1993;100(1):124S-7S.

28. Sontheimer RD. Would a new name hasten the acceptance of amyopathic dermatomyositis (dermatomyositis siné myositis) as a distinctive subset within the idiopathic inflammatory dermatomyopathies spectrum of clinical illness? J Am Acad Dermatol. 2002;46(4):626-36.

29. Singer M, Deutschman CS, Seymour CW, Shankar-Hari M, Annane D, Bauer $M$, et al. The third international consensus definitions for sepsis and septic shock (Sepsis-3). JAMA. 2016;315(8):801-10.

30. Brantly M, Avila NA, Shotelersuk V, Lucero C, Huizing M, Gahl WA. Pulmonary function and high-resolution $C T$ findings in patients with an inherited form of pulmonary fibrosis, Hermansky-Pudlak syndrome, due to mutations in HPS-1. Chest. 2000;117(1):129-36.

31. Terriff BA, Kwan SY, Chan-Yeung MM, Müller NL. Fibrosing alveolitis: chest radiography and $C T$ as predictors of clinical and functional impairment at follow-up in 26 patients. Radiology. 1992;184(2):445-9.

32. Ichikado K, Suga M, Muranaka H, Gushima Y, Miyakawa H, Tsubamoto M, et al. Prediction of prognosis for acute respiratory distress syndrome with thin-section CT: validation in 44 cases. Radiology. 2006;238(1):321-9.

33. Yamagata K, Li X, Ikegaki S, Oneyama C, Okada M, Nishita M, et al. Dissection of Wnt5a-Ror2 signaling leading to matrix metalloproteinase (MMP-13) expression. J Biol Chem. 2012;287(2):1588-99.

34. Satoh $M, A C M H, E K L C$. Immunodiagnosis of autoimmune myopathies. In: B D RGH, JL S, editors. Manual of molecular and clinical laboratory immunology, eighth edition. 8th ed. Washington, D. C: ASM Press; 2016. p. 878-87.

35. Ceribelli A, Fredi M, Taraborelli M, Cavazzana I, Tincani A, Selmi C, et al. Prevalence and clinical significance of anti-MDA5 antibodies in European patients with polymyositis/dermatomyositis. Clin Exp Rheumatol. 2014;32(6):891-7.

36. Nara M, Komatsuda A, Omokawa A, Togashi M, Okuyama S, Sawada K, et al. Serum interleukin 6 levels as a useful prognostic predictor of clinically amyopathic dermatomyositis with rapidly progressive interstitial lung disease. Mod Rheumatol. 2014;24(4):633-6.
37. Yasuda H, Ikeda T, Hamaguchi Y, Furukawa F. Clinically amyopathic dermatomyositis with rapidly progressive interstitial pneumonia: the relation between the disease activity and the serum interleukin-6 level. J Dermatol. 2017:44(10):1164-7.

38. Grom AA, Mellins ED. Macrophage activation syndrome: advances towards understanding pathogenesis. Curr Opin Rheumatol. 2010;22(5):561-6.

39. Kumakura S, Ishikura H, Kondo M, Murakawa Y, Masuda J, Kobayashi S. Autoimmune-associated hemophagocytic syndrome. Mod Rheumatol. 2004; 14(3):205-15.

40. Bracaglia C, de Graaf K, Pires Marafon D, Guilhot F, Ferlin W, Prencipe G, et al. Elevated circulating levels of interferon- $\gamma$ and interferon- $\gamma$-induced chemokines characterise patients with macrophage activation syndrome complicating systemic juvenile idiopathic arthritis. Ann Rheum Dis. 2017; 76(1):166-72.

41. Kumakura S, Murakawa Y. Clinical characteristics and treatment outcomes of autoimmune-associated hemophagocytic syndrome in adults. Arthritis Rheumatol. 2014:66(8):2297-307.

42. Gono T, Kawaguchi Y, Ozeki E, Ota Y, Satoh T, Kuwana M, et al. Serum ferritin correlates with activity of anti-MDA5 antibody-associated acute interstitial lung disease as a complication of dermatomyositis. Mod Rheumatol. 2011;21(2):223-7.

43. Hoshino K, Muro Y, Sugiura K, Tomita Y, Nakashima R, Mimori T. Anti-MDA5 and anti-TIF1-gamma antibodies have clinical significance for patients with dermatomyositis. Rheumatology (Oxford). 2010;49(9):1726-33.

44. Bilgic H, Ytterberg SR, Amin S, McNallan KT, Wilson JC, Koeuth T, et al. Interleukin-6 and type I interferon-regulated genes and chemokines mark disease activity in dermatomyositis. Arthritis Rheum. 2009;60(11):3436-46.

45. Narazaki M, Hagihara K, Shima Y, Ogata A, Kishimoto T, Tanaka T. Therapeutic effect of tocilizumab on two patients with polymyositis. Rheumatology (Oxford). 2011;50(7):1344-6.

\section{Ready to submit your research? Choose BMC and benefit from:}

- fast, convenient online submission

- thorough peer review by experienced researchers in your field

- rapid publication on acceptance

- support for research data, including large and complex data types

- gold Open Access which fosters wider collaboration and increased citations

- maximum visibility for your research: over $100 \mathrm{M}$ website views per year

At BMC, research is always in progress.

Learn more biomedcentral.com/submissions 